\title{
NOTES ON A CASE OF POISONING BY CORROSIVE SUBLIMATE.
}

\author{
By GIBBON FITZGIBBON, M.D., F.R.C.P.I.;
}

Gynæcologist to the Royal City of Dublin Hospital.

[Read in the Section of Obstetrics, November 30, 1917.]

I HAVE met only one case like the following reported in the medical literature, but it was similar in almost every respect, the poison having been introduced in the same way and, although coming under treatment in about eight hours, the patient died on the ninth or tenth day.

The patient, a married woman, aged 32 years, 3-para, introduced an 8.75 grain tabloid of corrosive sublimate into her vagina to prevent possible impregnation. Four hours later I was sent for owing to acute pain in the vagina. Fairly profuse watery discharge issued from the vagina, and the whole mucous membrane was considerably reddened; there was no ulceration or evident break in the surface, the vagina was very dry and hot, with very marked tenderness. No remains of the tabloid were found. I gave a very copious and prolonged douche of soapy water, and told the patient to drink as much fluid as possible.

Next morning (after 15 hours) patient appeared very well; there was increased tenderness in the vagina, and necrosis of the surface epithelium. Secretion of urine was plentiful and the bowels had acted well. ("House mixture " given previous night). Another prolonged douche was given and a large rubber tube introduced into the 
vagina in view of the inevitable sloughing. During the next twelve hours the bowels acted five times, plenty of urine was secreted and large quantities of water, milk and soda water, and albumen water were taken, also light diet.

After 36 hours : condition of patient seemed very good, the vaginal mucous membrane had become necrotic, slight swelling of the gums was evident and a distinct blue line along the margins. The tongue felt sore, with general feeling of dryness in the throat. The ducts of the submaxillary glands were both marked by small patches of white. During the last twelve hours only about five ounces of urine were secreted. In the next twenty-four hours about two ounces. The bowels acted twice after purgation, and quantities of fluid were taken by the mouth. Hot fomentations were continually applied over the kidneys, and a mixture of digitalis, strychnine and spirit of nitrous ether was given hourly.

On the third morning (60 hours) the whole vagina was lined with a dark brown necrotic coating, the edges of the gums along the teeth had become necrotic, the patches under the tongue had spread considerably and become necrotic. The whole tongue was much swollen and there was difficulty in swallowing. During the next twenty-four hours less fluid was taken, so saline enemata were started (every three hours 8 to 10 ounces). Not more than a few drachms of urine were secreted.

Fourth morning (84 hours). There was little change, more necrosis in the mouth and more difficulty in swallowing, but during the day about 80 ounces of fluid were taken and the saline enemata all retained but one. No urine; temperature continued normal; pulse about 80 and of good volume. 
80 Notes on a Case of Poisoning by Corrosive Sublimate.

Fifth day (108 hours). Conditions much the same; about twenty ounces of fluid were taken; and enemata were fairly well retained. Action of the bowels more frequent (six motions in twenty-four hours). Motions watery, with what appeared to be flaky mucus or bits of necrotic mucous membrane and fæcal matter. Urine secreted about one and a half ounces.

Sixth morning (132 hours). Mouth very bad, a lot of sloughs beginning to loosen; patient unable to speak owing to swelling of tongue and throat. Only about twenty ounces of fluid taken. During the day enemata well retained and bowels acted once. Sulphur bath given. No urine. During the following night only three ounces of milk taken and the enemata had to be reduced to four ounces. Only two were retained. There was one motion of the bowels containing blood. Temperature still normal. Pulse 80-90.

Seventh morning (156 hours). Patient quite unable to swallow. Very little given by enemata. Esophageal tube passed three times and two pints of sodium bicarb. solution introduced, with half-pint of milk and egg-flip on each occasion. There were five motions, the first containing much melæna and others an increasing quantity of fresh blood. In the evening one ounce of urine was drawn off.

Eighth morning (180 hours). Condition much the same. During the day only two motions, rather less blood, though marked and fresh. In the morning one and three quarter ounces of urine by catheter; three ounces in evening. Esophageal tube passed twice, and six pints of fluid and egg-flip given. Patient slept a little more and seemed easier, and able to swallow a little.

Ninth morning (204 hours). Patient very drowsy. During the day blood was passed several times from 
the bowels, and she became somewhat delirious. Horse serum $20 \mathrm{c.cm}$. given hypodermically. During the twentyfour hours eleven ounces of urine were drawn off and six pints of fluid given by tube. Temperature still normal; pulse 80-90. During the night thirteen ounces of urine drawn off. Patient less restless though did not sleep soundly. There was a good deal of bleeding from the bowel. Horse serum $20 \mathrm{c.cm}$. repeated. Pulse about 80.

Tenth moming (228 hours). Patient more drowsy and respiration heavier; twitchings of mouth and face. The temperature rose to $101^{\circ} \mathrm{F}$.; pulse about 90 . Six pints of fluid given by œsophageal tube. The bleeding from the bowel more profuse. At 10.30 a.m. eighteen ounces of urine drawn off, and at 6 p.m. twenty-two ounces. During the night the patient became semi-comatose. A large quantity of blood passed per rectum; twenty-seven ounces of urine drawn off. Numerous slight fits occurred with marked twitchings of the face and sometimes arms, but no stage of rigidity. Temperature rose gradually to $102^{\circ}$ and pulse to 104 .

Eleventh morning (252 hours). Patient unconscious; bleeding from bowel continuing; only five ounces of urine drawn off at 10 a.m. and four ounces at 3.40 p.m. Esophageal tube passed with difficulty owing to convulsive spasm, and four pints of fluid introduced. Temperature about $102^{\circ}$. The patient died at 4.45 p.m. From the beginning of the suppression of urine the patient was kept surrounded with hot water bottles and very free sweating was maintained.

Post-mortem.-On opening the abdomen the intestines were found slightly injected; nothing very remarkable was 
noticed on superficial examination, no ascitic fluid. The upper part of the jejunum had enlarged blood vessels on outside; mucous membrane normal. The mucous membranes of the ileum and upper part of the colon also showed no very marked change, but in the lower part of the colon and sigmoid flexure the mucous membrane was found detached and came off in patches. The kidneys were very slightly enlarged and very congested on surface, some patches softer than the organs in general. Liver little if anything altered. Microscopic sections of large intestine showed loss of surface mucous membrane. Sections of the kidneys showed almost universal destruction of epithelium, particularly marked in tubules, many being quite devoid of epithelium. No extravasation of blood in excretory tubules or in glomeruli.

When urine began to be secreted again on the eighth day it was pale in colour and contained only a very slight trace of albumen; at no time on subsequent days was there any blood in the urine or more than a mere trace of albumen. A small quantity of urine at this time was tested for evidence of metallic mercury but none was found. On one day during the period of practically complete suppression of urine what came away in the catheter was blood-stained, but this may have come from the urethra.

The sloughing of the vagina was very deep and the sloughs never separated; there always appeared to be sloughing deeper in the tissues. There was relatively little bleeding from the vagina.

When the kidneys began to act again it was hoped that the elimination of the poison would be effected, but from that time the symptoms of uræmic poisoning began to 
develop and increased, although during the three days prior to death the quantity of fluid excreted by the kidneys steadily increased, being eleven ounces, forty-two ounces, and fifty-four ounces, falling off to nine ounces in the last eight hours.

In reporting my case I am not able to lay down any proper line of treatment to be adopted; that which I did adopt was not successful. I endeavoured to promote. excretion, but it appears that the excretion of the poison had so destructive an effect upon the epithelium of the kidneys that it destroyed their normal action, and the patient died from renal insufficiency and consequent uræmia. Also it must have been the excretion of the poison which caused the necrosis of the mucous membrane of the lower bowel and of the salivary glands and gums. This latter was evident in thirty-six hours, and progressed very rapidly. The effect upon the kidneys showed itself after twenty-four hours, and from twelve hours later, during the next seven days, only about three ounces of urine were drawn off from the bladder. Unfortunately no full analysis of the fluid excreted by the kidneys was made, but it is probable that if such had been done the fluid would have been found to consist almost solely of the watery elements of urine with little or no urea and other waste matters, and thus account for the terminal uræmia in spite of the apparent recovery of the renal function.

It appears to me that the only means of combating the poison in such a case would be to tie up the mineral in the tissues, so that it would not be excreted in bulk, and this must be done very soon after its introduction; but how this is to be done or with what drug I do not know. 
81 Notes on a Case of Poisoning by Corrosive Sublimate.

Possibly an extensive and deep removal of the mucous membrane of the whole vagina would be the only means of removing the poison before its absorption into the system, and so prevening its excretion by the kidneys and other organs upon which it has so destructive an action.

Since reading the above paper I have received the following reprints:- "Calcium Sulphide as the Chemical and Clinical Antidote for Mercuric Chloride Poisoning, with Experiments and Case Reports," Journal Tech. and Clinl. Med., Vol. II., No.7, in which are reported experiments upon dogs and rabbits and several cases of poisoning of humans. With the dogs, where doses of 7.5 grs. hydrag. perchlor. were given, death followed in one to four days. The lethal dose was found to be .25 grains per pound weight of animal. The antidote used was an aqueous solution of calcium sulphide, 1 grain to the ounce, and given in the proportion of 1 grain for each grain of the hydrag. perchlor. administered intravenously. When this was done, 48 hours after given the poison the dogs rapidly recovered, and when killed subsequently were found normal. In two experiments old calcium sulphide solution was used, and death followed, which was shown to be due to the loss of sulphur in the old solutions.

Several cases of poisoning in human beings are also reported, with perfect curative results, where 7.5 grains of hydrag. perchlor. had been taken, and the antidote administered as late as 15 hours' after, either intravenously or in 2 to 5 grain goses by mouth, given hourly, until an excessive amount is taken. The solution of calcium sulphide must be fresh, and there is danger with old solutions owing to the free action of calcium. The experiments and cases appear to be absolutely conclusive. 
"Case of Malignant Syphilis Treated by Intensive Mercurialisation," Weekly Bulletin, Acad. Med., Cincinnatt, Vol. III., No. 26. The patient, aged 5 years, weight $25 \mathrm{lbs}$, was given on March 6th, 7th and 9th 2 grains of hydrag. perchlor., and four hours later 2 grains of calcium sulphide, repeated hourly for three doses. No effect of poisoning. This treatment was carried out on eleven subsequent occasions without any evidence of mercurial poisoning.

Dr. KirkPatrick suggested that it was possible to treat the case by repeated bleedings and intravenous salines. He thought that the attempt to make the kidneys secrete was possibly harmful, because of the danger of excreting by the kidneys, which is great. $\mathrm{He}$ thought sweating would be beneficial.

Dr. O'FARRELL asked if the woman swallowed some of the corrosive as well.

$D_{R}$. Ashe considered that, if the vagina was plugged with tampons soaked in ordinary lime water in such a ceses. the solid would become inert. 